not too unusual among Gadwall (J. B. Gollop). Widgeon (Mareca americana): May 18 north of Floral; one with tinges of yellow and darker flecks; it was with a flock of four other widgeon (M. A. Gollop). Stilt Sandpiper (Micropalama himantopus): on May 24 at Proctor Lake; one individual in a flock had a dirty white head and neck. Brewer's Blackbird (Euphagus cyanocephalus): on May 8 near CFQCTV tower; the female of a pair had one outer and one other tail feather pure white. There were three or four small spots of white on the bird's body. (J. B. Gollop.)

Other records: Horned Lark (Eremophila alpestris): A pair nested within the city limits in a newly planted lawn at 423 Quance Avenue. Three young were fledged. (J. F. Roy, R. V. Folker). Black-billed Magpie (Pica pica: a flock that centered its activities around a turkey farm just south of the city limits was checked almost weekly for four months. On August 1, there were at least 74 birds. The peak number was $114+$ on August 26. There were $93+$ on September 8 , $45+$ on October $17,42+$ on November 7 and $22+$ on November 22 , the last date that a count was made before the road became blocked with snow. Most satisfactory counts were made as the birds flew about a mile south of the farm to roost after sunset (J. B. Gollop). Lark Bunting (Calamospiza melanocorys): Since the birds reported by Roy (1960), which omitted a male near Bradwell (June 11, 1960), our records are as follows: 1961: four birds, south of Aberdeen and west of Vonda (May 21); 1962: none; 1963: 13 birds, Burke Lake (May 25 and June 2), Proctor Lake and Dundurn (June 16), Laura and Delisle (July 20), Dundurn (September 2); 1964: 3 individuals, Floral (May 18) and Hanley (June 21).

\section{LITERATURE CITED}

Gollop, J. B., J. F. Roy, and R. V. Folker. 1963. Some 1962 bird records for the Saskatoon district. Blue Jay, 21 (1): 12-13.

Roy, F. 1960. Birds of special interest in the Saskatoon area, Spring, 1960. Blue Jay, 18 (3): $117-118$.

\title{
May Day Count, Saskatoon Natural History Society
}

May 23, 1964: A species count within a circle centering on Saskatoon and having a radius of 25 miles. 5:00 a.m. to $8: 30$ p.m., CST. 20 observers in four parties. Temp. $70^{\circ}$, strong winds after 12 noon. Total, 129 species.

Of particular interest are the nesting record of the Ferruginous Hawk and the first Saskatoon record of the Parasitic Jaeger. The peak of the warbler migration occurred about a week prior to the count.

Common Loon, Red-necked Grebe, Horned Grebe, Eared Grebe, Western Grebe, Piedbilled Grebe, Great Blue Heron (nests with eggs), American Bittern, Canada Goose (pair with young at north end of Pike Lake), Mallard, Gadwall, Pintail, Green-winged Teal, Blue-winged Teal, American Widgeon, Shoveler, Redhead, Ring-necked Duck, Canvasback, Lesser Scaup, Common Goldeneye, Bufflehead, White-winged Scoter, Ruddy Duck, Sharp-shinned Hawk, Red-tailed Hawk, Swainson's Howk, Ferruginous Hawk (nest with eggs), Marsh Hawk, Pigeon Hawk, Sparrow Hawk, Ruffed Grouse, Sharp-tailed Grouse, Ring-necked Pheasant, Gray Partridge, Sora, American Coot, Semipalmated Plover, Killdeer, Golden Plover, Black-bellied Plover, Common Snipe, Upland Plover, Spotted Sandpiper, Willer, Lesser Yellowlegs, Pectoral Sand- piper, Baird's Sandpiper, Dowitcher (Longbilled?) Stilt Sandpiper, Semipalmated Sandpiper, Marbled Godwit, Sanderling, American Avocet, Wilson's.'Phalarope, Northern Phalarope, PARASITIC JAEGER (first Saskatoon record, B. Gollop), California Gull, Ring-billed Gull, Franklin's Gull, Common Tern, Black Tern, Rock Dove, Mourning Dove, Great Horned Owl, Burrowing Owl, Short-eared Owl, Belted Kingfisher, Yellowshafted Flicker, Yellow-bellied Sapsucker, Downy Woodpecker, Eastern Kingbird, Western Kingbird, Eastern Phoebe, Least Flycatcher, Horned Lark, Tree Swallow, Bank Swallow, Barn Swallow, Cliff Swallow, Blue Jay, Blackbilled Magpie, Common Crow, Black-capped Chickadee, House Wren, Long-billed Marsh Wren, Catbird, Brown Thrasher, Robin, Swainson's Thrush, Veery, Mountain Bluebird, Water Pipit, Sprague's Pipit, Loggerhead Shrike, Starling, Red-eyed Vireo, Philadelphia Vireo, Warbling Vireo, Tennessee Warbler, Yellow Warbler, Blackpoll Warbler, Ovenbird, Yellowthroat, American Redstart, House Sparrow, Bobolink, Western Meadowlark, Yellow-headed Blackbird, Red-winged Blackbird, Baltimore Oriole, Brewer's Blackbird. Common Grackle, Brown-headed Cowbird, Rosebreasted Grosbeak, Pine Siskin, American Goldfinch, Red Crossbill, Rufous-sided Towhee, Savannah Sparrow, Sharp-tailed Sporrow, Vesper Sparrow, Tree Sparrow (?), Chipping Sparrow, Clay-colored Sparrow, White-throated Sparrow, Song Sparrow, Lapland Longspur, Chestnut-collared Longspur. - Frank Roy, compiler. 